\title{
Specific copy number changes as potential predictive markers for adjuvant chemotherapy in non-small cell lung cancer
}

\author{
Mitsuo Sato \\ Department of Pathophysiological Laboratory Sciences, Nagoya University Graduate School of Medicine, Nagoya, Japan \\ Correspondence to: Mitsuo Sato. Department of Pathophysiological Laboratory Sciences, Nagoya University Graduate School of Medicine, 1-1-20 \\ Daiko-minami, Higashi-ku, Nagoya 461-8673, Japan. Email: msato@met.nagoya-u.ac.jp. \\ Comment on: Rotolo F, Zhu CQ, Brambilla E, et al. Genome-wide copy number analyses of samples from LACE-Bio project identify novel prognostic \\ and predictive markers in early stage non-small cell lung cancer. Transl Lung Cancer Res 2018;7:416-27.
}

Submitted Nov 13, 2018. Accepted for publication Nov 21, 2018.

doi: $10.21037 /$ tlcr.2018.11.04

View this article at: http://dx.doi.org/10.21037/tlcr.2018.11.04

Structural and numerical changes in cancer genome have been extensively investigated because of their fundamental contribution to initiation and development of human cancer. In the past, conventional karyotypic analysis of tumor samples led to identification of several oncogenes and tumor suppressor genes, such as the $M Y C$ and $R B(1,2)$. Over the past two decades, gene copy analysis technology has progressed remarkably, enabling the identification of regions recurrently gained or lost, with a size small enough to narrow down to genes that are potentially relevant for the malignant properties of tumor cells. For example, TITF1, and SOX2 have been identified as novel oncogenes through the integrative analysis of genome-wide copy number and gene function $(3,4)$. Such genes may serve as therapeutic targets, thereby directly contributing to clinical practice. Another way to translate copy number data into the clinic is to utilize them as prognostic and predictive markers. In particular, developing predictive markers for certain treatments is vital because they make it possible to maximize the therapeutic benefits to patients.

Adjuvant chemotherapy (ACT) for patients with nonsmall cell lung cancer (NSCLC) underdoing complete resection has become the standard treatment based on several independent large-scale randomized studies (5). The studies reproducibly showed that platinum doublet treatments produce approximately $4-15 \%$ survival benefit. This benefit is very meaningful because it may directly contribute to cure of the disease. However, the treatments are inevitably associated with adverse events, which causes approximately $1 \%$ of deaths. Thus, establishing reliable biomarkers for
ACT is very important. Both ERCC1 and RRM1 emerged as promising biomarkers for ACT $(6,7)$; however, their usefulness has not yet been demonstrated by a phase III trial (8). Therefore, predictive biomarkers for ACT that can readily be used in the clinical settings are currently lacking (9).

Recently, Rotolo et al. have reported a comprehensive study in Translational Lung Cancer Research evaluating copy number changes using samples from the Lung Adjuvant Cisplatin Evaluation Biomarker (LACE-Bio) project, which was launched to identify promising biomarkers for ACT in a large cohort of patients participating in the ACT trials; the IALT, ANITA, JBR10, and CALGB9633 (10). The authors analyzed copy number alterations in 976 formalin-fixed paraffin-embedded (FFPE) samples with over 200 thousand single nucleotide polymorphism (SNP) probes. This resulted in the identification of recurrently amplified or lost regions with high resolution, followed by the correlation between those regions and the clinical outcome with a median follow-up duration of 5.2 years. The authors have confirmed several recurrently gained or lost regions, defined as 2-fold higher or lower copy number. For instance, approximately $40 \%$ of the samples exhibited loss of $C D K N 2 A$ and $C D K N 2 B$ residing adjacently in $9 \mathrm{p} 21.3$. $C D K N 2 A$ is alternatively transcribed, encoding two critical tumor suppressor proteins: p16INK4 and p14ARF (11). CDKN2B encodes p15, another cell cycle inhibitor (12). Many studies have shown that alterations in these genes, including copy number loss, are associated with a poor prognosis in NSCLC patients $(13,14)$. In addition, the authors found that $L K B 1$ (also known as serine/ 
threonine kinase 11; STK11) is prognostic. LKB1 functions as a multifunctional tumor suppressor gene that regulates cell polarity, metabolism, proliferation, and migration and is frequently mutated, particularly in lung adenocarcinoma (15). Its loss is associated with poor prognosis in various types of human cancer (16), demonstrating its role in tumor suppression. Thus, Rotolo et al. successfully confirmed the prognostic relevance of these regions.

In addition to these previously reported genes, the study found several novel candidates as predictors for prognosis. Nevertheless, prognostic values of some of them seem contradictory to previously reported findings. For example, the guanine nucleotide exchange factor gene VAV1 in 19p13.3-2, initially identified as a proto-oncogene through NIH3T3 cell screen, was conversely reported to be not amplified but lost in the study, correlating with worse prognosis. A recently study demonstrated the oncogenic functions of $V A V 1$, showing that three somatic gene mutations occurring in human lung adenocarcinoma exhibit transformation ability, as measured by anchorage independent growth and tumor formation in immunodeficient mice (17). In addition, the lost region in 19p13.3-2 contains another oncogenic gene, TCF3 (18). Therefore, further studies are needed to evaluate prognostic potential of genes in this region.

The strength of that study is that the samples were obtained from a randomized study evaluating the impact of ACT. This allowed exploration of altered regions that are potential predictive markers for ACT efficacy. The authors identified three regions that correlated with survival benefit in patients receiving ACT. Among them, an amplified region in $20 \mathrm{q} 11.21$ is interesting because it includes multiple genes potentially involved in oncogenesis and chemotherapy response. A previous study found that this region was amplified in NSCLC (19). Among these genes, TPX2, a cofactor of Aurora Kinase A (AURKA), plays a pivotal role in regulating microtubule assembly during mitosis (20), thereby potentially affecting the efficacy of microtubule-targeted drugs, such as vinorelbine and paclitaxel. Because most ACTtreated patients participating in the LACE-Bio project received vinorelbine or paclitaxel-containing platinum doublets, it is speculated that an increased copy number of TPX2 are associated with ACT efficacy. Recently, Orth et al. demonstrated that TPX2 expression is involved in the radiosensitizing effect of paclitaxel in vitro (21). The study also reported that in The Cancer Genome Atlas (TCGA) cohort treated with taxane-based radiochemotherapy,
AURKA and TPX2 levels were associated with better overall survival in lung adenocarcinoma. Nevertheless, the results of Orth et al. contradict those of Rotolo et al. because they found that a high TPX2 expression correlates with better survival in taxane-based radiochemotherapy and patients with an increased copy number of TPX2 had worse benefits from ATC. However, both studies differ significantly in terms of treatment settings (ACT vs. radiochemotherapy). Thus, the status of targeted tumors cells differed significantly; ACT mostly targeted micrometastatic lesions with many dormant tumor cells whereas radiochemotherapy mostly targeted massive primary lesion with many actively proliferating tumor cells. For this reason, it is possible that an increased TPX2 expression affected the benefits of each treatment in different ways. Therefore, further validation of these results is required.

The amplified region of $20 \mathrm{q} 11.21$ contains other genes with oncogenic properties including BCL2L1 and PDRG1. Multiple coamplified genes may exhibit oncogenic properties synergistically (22). It is therefore possible that two or more gens co-amplified in $20 \mathrm{q} 11.21$ synergistically contribute to tumor development and/or affect sensitivity to chemotherapy. To investigate these hypotheses, further studies are needed to evaluate the effects of the introduction of putative target genes in 20q11.21 alone or in combination on oncogenic transformation or chemosensitivity using a normal lung epithelial model. An HBEC model developed by our group could be one of the ideal models because it has been extensively used to evaluate the effect of introducing or silencing of multiple genes on oncogenic properties or chemosensitivity (23-25).

In conclusion, the study by Rotolo et al. suggests several specific gained or lost regions in NSCLC samples, which could potentially serve as prognostic biomarkers and/or predictors for ACT. Further evaluation of their usefulness in different sets of cohorts as well as biological analyses are needed to proceed to prospective phase III studies investigating the prognostic value and predictive roles in ACT of specific copy number changes.

\section{Acknowledgements}

Funding: This work was supported, in part, by a Grant-inAid for Scientific Research (B) $18 \mathrm{H} 02819$ for M Sato from the Japan Society for the Promotion of Science.

\section{Footnote}

Conflicts of Interest: The author has no conflicts of interest to 
declare.

\section{References}

1. Little CD, Nau MM, Carney DN, et al. Amplification and expression of the c-myc oncogene in human lung cancer cell lines. Nature 1983;306:194-6.

2. Friend SH, Bernards R, Rogelj S, et al. A human DNA segment with properties of the gene that predisposes to retinoblastoma and osteosarcoma. Nature 1986;323:643-6.

3. Bass AJ, Watanabe $\mathrm{H}$, Mermel CH, et al. SOX2 is an amplified lineage-survival oncogene in lung and esophageal squamous cell carcinomas. Nat Genet 2009;41:1238-42.

4. Weir BA, Woo MS, Getz G, et al. Characterizing the cancer genome in lung adenocarcinoma. Nature 2007;450:893-8.

5. Pirker R. Adjuvant chemotherapy in patients with completely resected non-small cell lung cancer. Transl Lung Cancer Res 2014;3:305-10.

6. Olaussen KA, Dunant A, Fouret P, et al. DNA repair by ERCC1 in non-small-cell lung cancer and cisplatin-based adjuvant chemotherapy. N Engl J Med 2006;355:983-91.

7. Simon G, Sharma A, Li X, et al. Feasibility and efficacy of molecular analysis-directed individualized therapy in advanced non-small-cell lung cancer. J Clin Oncol 2007;25:2741-6.

8. Bepler G, Williams C, Schell MJ, et al. Randomized international phase III trial of ERCC1 and RRM1 expression-based chemotherapy versus gemcitabine/ carboplatin in advanced non-small-cell lung cancer. J Clin Oncol 2013;31:2404-12.

9. Wallerek S, Sorensen JB. Biomarkers for efficacy of adjuvant chemotherapy following complete resection in NSCLC stages I-IIIA. Eur Respir Rev 2015;24:340-55.

10. Rotolo F, Zhu CQ, Brambilla E, et al. Genome-wide copy number analyses of samples from LACE-Bio project identify novel prognostic and predictive markers in early stage non-small cell lung cancer. Transl Lung Cancer Res 2018;7:416-27.

11. Kamijo T, Zindy F, Roussel MF, et al. Tumor suppression at the mouse INK4a locus mediated by the alternative reading frame product p19ARF. Cell 1997;91:649-59.

12. Hannon GJ, Beach D. p15INK4B is a potential effector of TGF-beta-induced cell cycle arrest. Nature 1994;371:257-61.

13. Jin $M$, Inoue $S$, Umemura $T$, et al. Cyclin D1, p16 and retinoblastoma gene product expression as a predictor for prognosis in non-small cell lung cancer at stages I and II.
Lung Cancer 2001;34:207-18.

14. Zhao Y, Li Y, Lu H, et al. Association of copy number loss of CDKN2B and PTCH1 with poor overall survival in patients with pulmonary squamous cell carcinoma. Clin Lung Cancer 2011;12:328-34.

15. Sanchez-Cespedes M, Parrella P, Esteller M, et al. Inactivation of LKB1/STK11 is a common event in adenocarcinomas of the lung. Cancer Res 2002;62:3659-62.

16. Xiao J, Zou Y, Chen X, et al. The Prognostic Value of Decreased LKB1 in Solid Tumors: A Meta-Analysis. PLoS One 2016;11:e0152674.

17. Shalom B, Farago M, Pikarsky E, et al. Vav1 mutations identified in human cancers give rise to different oncogenic phenotypes. Oncogenesis 2018;7:80.

18. Taniue K, Kurimoto A, Takeda Y, et al. ASBEL-TCF3 complex is required for the tumorigenicity of colorectal cancer cells. Proc Natl Acad Sci U S A 2016;113:12739-44.

19. Tonon G, Wong KK, Maulik G, et al. High-resolution genomic profiles of human lung cancer. Proc Natl Acad Sci U S A 2005;102:9625-30.

20. Asteriti IA, Rensen WM, Lindon C, et al. The Aurora-A/ TPX2 complex: a novel oncogenic holoenzyme? Biochim Biophys Acta 2010;1806:230-9.

21. Orth M, Unger K, Schoetz U, et al. Taxane-mediated radiosensitization derives from chromosomal missegregation on tripolar mitotic spindles orchestrated by AURKA and TPX2. Oncogene 2018;37:52-62.

22. Kendall J, Liu Q, Bakleh A, et al. Oncogenic cooperation and coamplification of developmental transcription factor genes in lung cancer. Proc Natl Acad Sci U S A 2007; 104:16663-8.

23. Kakumu T, Sato M, Goto D, et al. Identification of proteasomal catalytic subunit PSMA6 as a therapeutic target for lung cancer. Cancer Sci 2017;108:732-43.

24. Sato M, Larsen JE, Lee W, et al. Human lung epithelial cells progressed to malignancy through specific oncogenic manipulations. Mol Cancer Res 2013;11:638-50.

25. Sato M, Vaughan MB, Girard L, et al. Multiple oncogenic changes (K-RAS(V12), p53 knockdown, mutant EGFRs, p16 bypass, telomerase) are not sufficient to confer a full malignant phenotype on human bronchial epithelial cells. Cancer Res 2006;66:2116-28.

Cite this article as: Sato M. Specific copy number changes as potential predictive markers for adjuvant chemotherapy in nonsmall cell lung cancer. Transl Lung Cancer Res 2018;7(Suppl 4):S346-S348. doi: 10.21037/tlcr.2018.11.04 